\title{
Campus Guidance System for International Conferences Based on OpenStreetMap
}

\author{
Ricky Jacob ${ }^{1}$, Jianghua Zheng ${ }^{1}$, Błażej Ciepłuch ${ }^{1}$, Peter Mooney ${ }^{2}$, and Adam \\ C. Winstanley ${ }^{1}$ \\ ${ }^{1}$ Geotechnology Group,Department of Computer Science, National University of \\ Ireland Maynooth, Co. Kildare. Ireland. \\ 2 Environmental Research Centre, Environmental Protection Agency, Richview, \\ Clonskeagh, Dublin 14. Ireland \\ rjacob@cs.nuim.ie, jianghua.zheng@nuim.ie, \\ bciepluch@cs.nuim.ie, peter.mooney@nuim.ie, adam.winstanley@nuim.ie
}

\begin{abstract}
We present a web-based, multi-lingual, campus guidance system with emphasis on pedestrian navigation aimed at providing support for delegates attending International Conferences at the National University of Ireland Maynooth (NUIM) campus. A special campus guidance system could improve the logistics of the conference. Potentially such improvements could attract more delegates to the conference. The Cloudmade Web Map Lite API which uses OpenStreetMap has been used for creating this interface. The system generates shortest pedestrian paths using both outdoor pavements and indoor corridors between various buildings and points of interests (POI). For visual assistance in pedestrian navigation geotagged images are used along the path at certain points in the route, such as road intersections, when the user needs to get their orientation correct. The interface is available in both English and Chinese language.
\end{abstract}

Key words: Campus guidance system, Pedestrian navigation, Cloudmade, Maps API, OpenStreetMap, International conference, WebGIS, LBS, Multi-lingual

\section{Introduction}

Where is the conference dinner? Where are the conference sessions on? What is the best pub in town? These are all common questions asked by delegates when they are attending a conference. These type of queries are among a set of challenging navigation tasks for delegates attending conferences and meetings. Delegates often arrive to the location of a conference with only a short time to familiarise themselves with the campus, the local transportation system, and what the best features of the location are. This paper describes the development of a campus navigation system to assist delegates attending conferences at the NUIM campus. While the system has been initially developed for the NUIM campus and a specific conference it has been designed to extend to other conferences within the NUIM campus and beyond. 


\subsection{Existing Campus Guidance and Navigation Systems}

Many visitors and new students come to the university campus at NUIM every year. A campus guidance system is always a helpful tool to help those unfamiliar with the campus to orientate themselves and guide them around the campus. There are some information systems that have already been developed for this purpose. Most of these applications use commercial GIS components based on $\mathrm{COM} / \mathrm{COM}+$ technology. [1] used MapX to provide a campus navigation service of Wuhan University in China. [2], [3] utilize ArcIMS (ESRI Map Server) to develop a campus navigation prototype. [4] used SuperMap Objects to build a campus navigation system for Henan Polytechnic University in China. GIS components of the systems are commercial and their data is generally privately owned and without regular update. With the widespread use of Google Maps and Virtual Earth mapping it is now easy to obtain free mapping data. This has allowed developers to build Location Based Services (LBS) for environments such as university campuses. [5] was one the earliest groups that focused on campus navigation and guidance system using Google Maps. This has seen many such applications become available. [6] developed a campus information system based on Google Maps for Xinjiang University in China. The University College London [7] has created a campus navigation system built using Google maps API. Using Google Maps or Virtual Earth Map APIs some campus information applications can provide 3D services. [8] utilize VRML (Virtual Reality Modeling Language) to build campus navigation system in 3D environment. Tsinghua University, Industrial and Commercial University of Chongqing, Nantong University have all established "virtual campuses" for visitor guidance [9]. There are also some other campus navigation systems with a certain special functions or to demonstrate specific technologies. [10] discussed ubiquitous computing architecture of the eyeJOT, a new context-aware smart campus information system combining ambient wall-sized displays with location-aware, context-sensitive information sharing on mobile devices. [11] discussed Gippy, which is a campus GIS information system supported by P2P technology. [12] show their research on a Service Orientated Architecture for campus information modelling. Applications like these could very well be used for delegates attending the international conferences held on a university campus. However, the participants usually have different goals from other visitors and new students on campus. Participants require more specific information from a campus guidance system. Compared to general campus navigation or information systems these type of applications are relative few. One such example is [13] who discussed a simple prototype of such an application based on MapXtreme.

The last few years have seen an increased interest in the design of pedestrian navigation systems for mobile phones. Some Nokia phones now ship with Nokia Maps 2.0 which enhances pedestrian navigation and also provides city guides in a new improved interface. There has been considerable research carried out which evaluates various modes of pedestrian navigation feedback. The various modalities used for input or output are audio or voice feedback [14], textual turn by turn instructions, graphical map interface [15][16], image based navigation 
[17], haptic feedback [18] and multi-modal techniques [19] where more than one modality is used for providing navigational cues. In this paper we look at how we can use freely available Maps API to create a pedestrian navigation module within a campus guidance system.

\subsection{Using Maps API}

Integrated web applications or mashups are increasingly being used. Mashups bring together utilities of two or more applications into one common interface. Research shows that mapping mashups are the highest among all the mashups deployed. Various sites list and review the mashups people. One such site is [20]. In the recent years there has been an increase in the use of free Maps API provided by various web map services [21] [22] [23] [24] to create ones own map based web service using their interface and data along with some of the users own data. Some of the hugely popular and successful mashups like Kayak for hotels listing in US, and that for real estate listing Trulia have shown how with proper design and planning one can generate revenue using such freely available map APIs. When building such applications, the availability of a complete digital map data for the area of interest is an issue if the extent of the area is not within the limits of a major city. But when this is extended to smaller towns and cities the availability of reasonably good digital web-based maps is poor. In this paper we look at maps of the NUIM area across various web based map service providers like Microsoft's Bing Maps, Google Maps, Yahoo Maps and OpenStreetMap. We find that there is a sever lack of spatial data for our area of interest.

Open Street Map(OSM)[23] is a free atlas of the entire world. OSM is a knowledge crowdsourcing model that provides user-generated street maps. There has been various geo-wiki applications [25][26] that utilises user-generated content for its maps. However OSM is probably the most extensive and effective project currently under development [27]. OSM is a convenient tool to release user-generated street maps. It also provides basic GIS functions, such as zoom in/out, pan and feature information tools. It also provides an API offering higher level GIS functions such as a routing API and browser API for mobile phones to view OSM. Some recent applications have even developed 3D routing functionality based on OpenStreetMap, geovis of OSM-3D . org is an recent example. Since OpenStreetMap is has a free creative commons open source license model it allows a very flexibile model of access to the underlying spatial data for developers and the ability to use the various APIs. The most important is it provides good quality user-generated street maps efficiently. In our work, the OpenStreetMap for Maynooth is a rich spatial model with many point, line and polygon features. The NUIM campus has been mapped extensively to almost its entirety and thus used as the test bed for creating this prototype to demonstrate how we can bring together the capabilities of a personalized Pedestrian Navigation System using a Maps API with the OSM providing the mapping to create a campus guidance system.

In developing a systems such as this there is an emphasis on creating an system which can be reused and not limited to a one time use [28]. The objective 
of the present work is to provide a campus guidance system for the delegates attending the China-Ireland Conference on Informations and Communication Technologies 2009 [29]. However, it should be easily extendible to other conferences in NUIM and possibly in other university locations in Ireland. The CloudMade Web Maps Lite API [30] for routing has been used in this work as it is built on the OpenStreetMap database. The remainder of this paper is organised as follows. In the next section we discuss the creation of the OSM map the NUIM Campus and Maynooth Town. Then the need for a campus guidance system is discussed and followed by our proposed system. The scope for future work is discussed at the end of the paper.

\section{Creation of Map data}

OSM data collection for areas in and around Maynooth started in December 2008. A GPS device manufactured by Globalsat(Model: DG-100) was used for this propose. This device has gained popularity with the OSM community and the drivers for the Linux OS are also available. For reading stored GPS tracks and saving them on the computer the application called GPS Babel [31] was used. The first stage of data collection and mapping involved preparing the line features for roads and footpaths within the NUIM Campus. The more detailed NUIM Campus containing all the buildings and other POI was done using an aerial photograph of the campus. Using the GPS device the road network for the entire town of Maynooth and some of the surrounding hinterlands were mapped. A bicycle mounted with the GPS device in the front was used to travel across the small streets in and around the town of Maynooth. Travel by bicycle instead of on foot saved much time. While capturing the positions of POIs and other features of interest the attribute data for the same were also collected. For example the names of streets (both in English and Irish), the names of restaurants, shops, and also the house numbers in various localities. Various OSM users across Ireland are updating the OSM database and as of July 2009, the entire network of major roads and highways across Ireland has been mapped. It is noticeably that most of the cities in Ireland are mapped extensively in OSM. Some of the smaller towns are poorly represented with OSM at present. Figurereffig:CastleDawson shows a large residential area in Maynooth where details like street names and house numbers were captured.

\section{Campus Navigation System}

There are a number of requirements of a campus navigation system. These requirements are from the two sides of a conference: participants of conferences held on campus and the conference organisers themselves. From the organisers point of view there is the requirement that the campus navigation system for the conference can provide all the relevant information to the delegates in a simple easy to use interface. Many conferences are held in NUIM each year the availability of such a campus navigation system will be always useful. Rather 


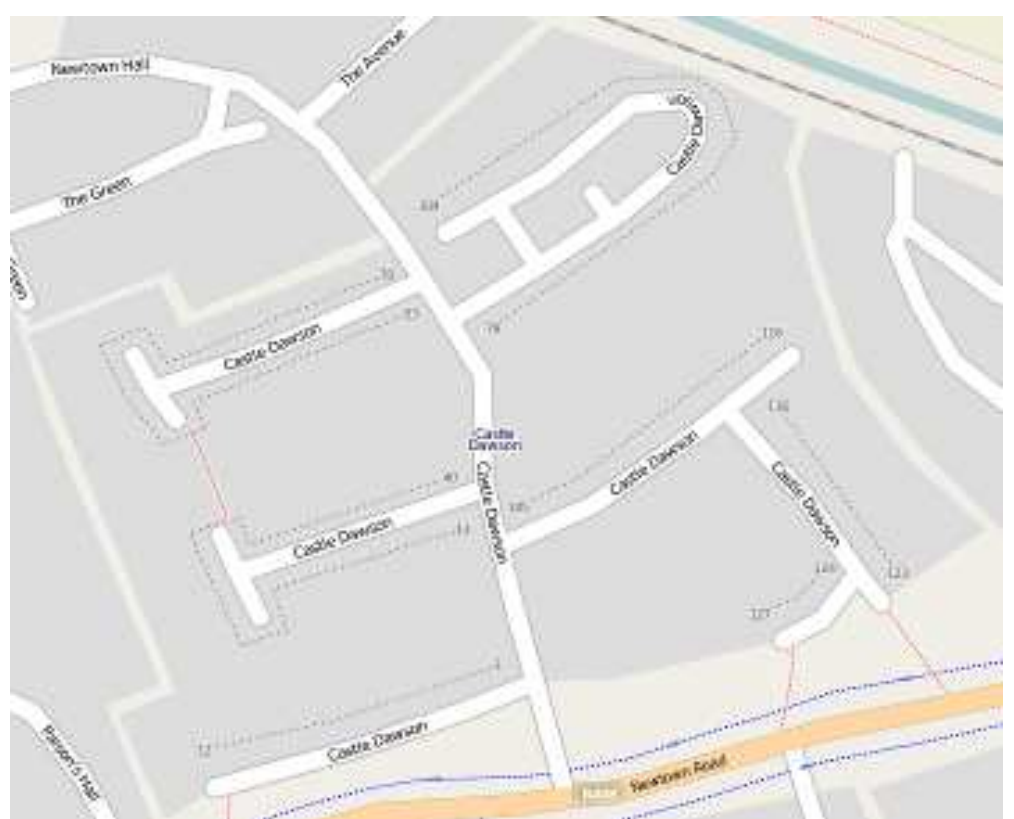

Fig. 1: An example of high resolution mapping in OSM. This is the Castle Dawson estate in Maynooth

than creating separate systems for each conference the ultimate goal is to have a standard campus navigation tool which can reused and can be easily customised by the conference organisers to satisfy their own specific requirements. After a brief survey of the academic and research staff in the Department of Computer Science at NUIM the most frequently asked questions of delegates of a conference are as follows:

- Where are the venues where the conference sessions will be held?

- How does one get to the conference venue?

- What are the various modes of transport available?

- What is the optimal route to get to from one location to another or from one POI to another POI?

- Where are the accommodation services?

- Where are the nearest restaurants that serves the food 'I' like?

- Where are the scenic spots around the campus which I can visit during the conference?

- Where are the locations of leisure activities on or near the conference location?

The first five in the list are potentially the most important questions for any conference delegate. The additional questions are of interest to delegates who wish to explore the campus and/or the surrounding town or locality. 


\subsection{Our Proposed Campus Navigation System}

The China-Ireland Conference has been used as a case study by using the CloudMade API to provide a Campus Navigation System with the inclusion of transportation related information and markers showing other POI within Maynooth Town. Initial we identified the various buildings and POI relevant to this conference. This list is based on the location of buildings where the sessions are held, hotels where the delegates will stay, the local pubs and restaurants, and the important transportation POI like train stations and bus stops [32]. Figure 2 shows a flowchart of the system architecture of the proposed campus navigation system.

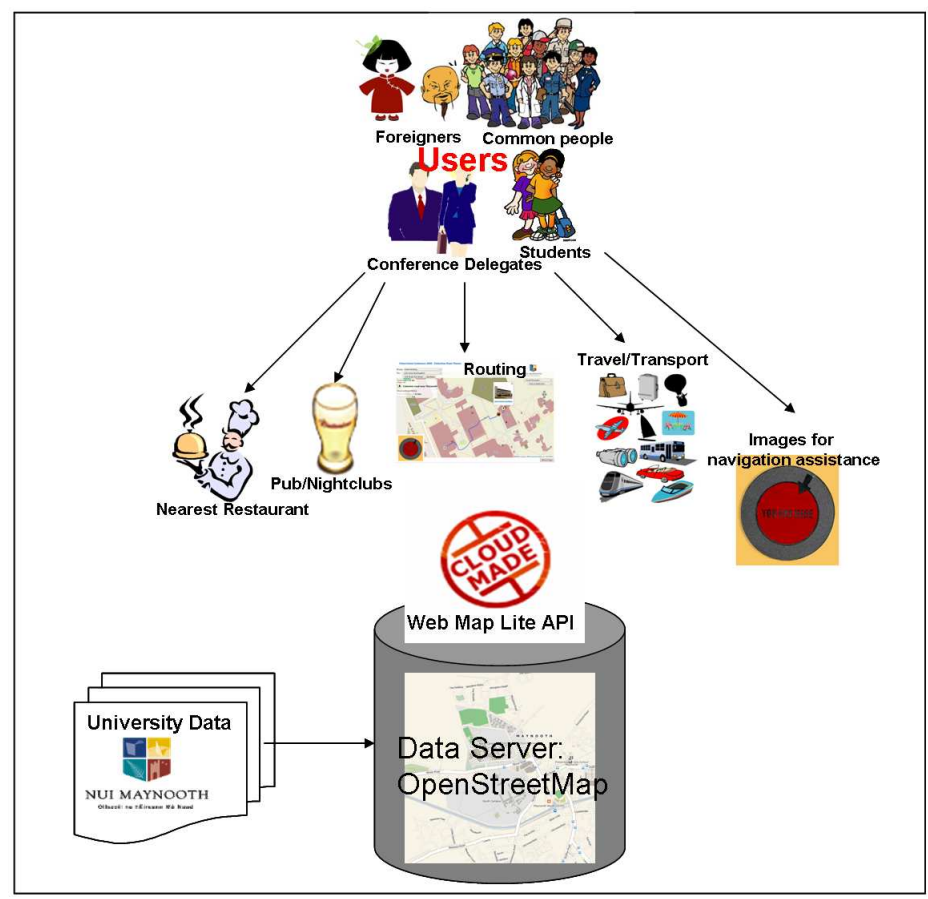

Fig. 2: A flowchart showing the system architecture for the campus navigation system

Routing Finding on the Campus Routing finding is the most important feature of this system. As stated above we use the CloudMade API to provide a turn-by-turn descriptions of shortest routes for car users, bicycle riders, and pedestrians. We have decided that we will only consider the case of pedestrian navigation around the campus. Most international delegates to the conference 
will not have access to an automobile or a bicycle during the duration of the conference. The richness of the OSM map of NUIM means that the CloudMade API can find routes on internal corridors and external footpaths, lanes, and streets. Figure 3 shows the shortest pedestrian path computed by the Cloudmade API between two buildings on the NUIM. The proposed route includes both indoor corridors and outdoor pedestrian pavements. The exact nature of the route is determined by the setting of the travelMode option as input to the route finding algorithm in the API. As mentioned above the choices available for travelMode are: $\{$ car, car/shortest, foot, bicycle $\}$.

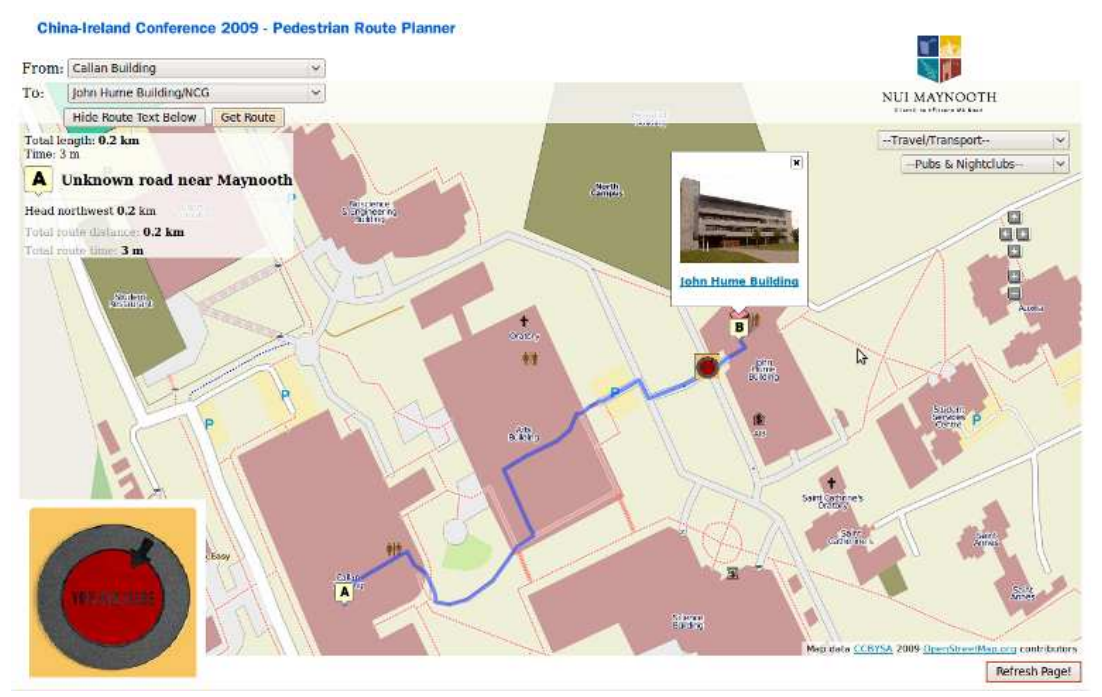

Fig. 3: The shortest pedestrian path between Callan and John Hume building using both internal corridors and external pavements

Geotagged Images for navigation Fourteen buildings and POI were identified in and around the campus that would be of relevance to the delegates attending the conference. For people new to an area the use of landmarks and geotagged images for navigation is of great assistance [33]. Figurereffig:geotagg shows how the route description is supplemented with the insertion of geotagged images using markers at intersections or places where the user needs to change direction. These visual clues will help the user with orientation and assisting in their sense of direction when taking the suggested route. Figure 5 shows the use of public transport travel information being provided to the user if it is applicable from their chosen route. The system allows users to plan shortest routes between the specified set of buildings and POI. Users can also drag a marker and place it at any point on the campus from which they want to generate a 
shortest path from. The system dynamically calculates the new shortest path and updates the map display accordingly. The geographical area from which the user can select a random points from which to plan new shortest paths between has been constrained to a $3 \mathrm{KM}$ grid centered on the campus.

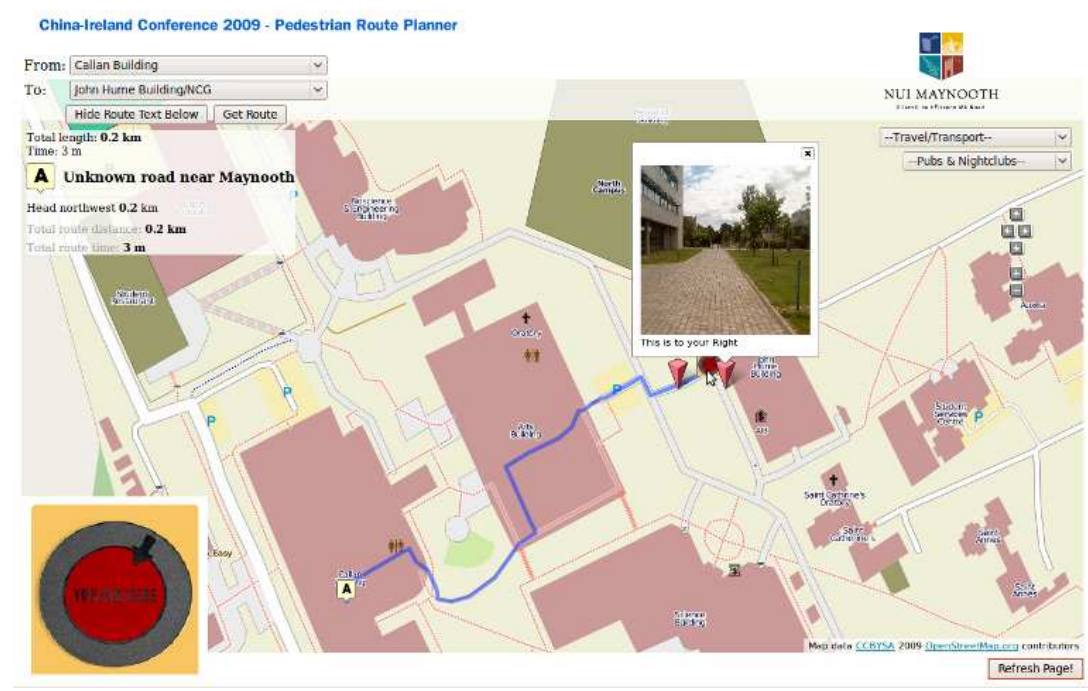

Fig. 4: Geotagged images used for assisting navigation

Travel and transport for the conference Sometimes when visiting a conference location understanding the local public transportation system can be a difficult task for strangers to the area. There is always a need to provide the user with travel and transport related information. Figure 5 shows the use of markers at various transit points and information and suggestion are provided to the user in the information window. This provides users with the option to view information on how to travel from the NUIM campus to the nearby city of Dublin. The information provided includes fares, timetables and links to all the important websites that he/she needs for using the public transportation in the area. This feature should save users a lot of time by providing them with targeted local information.

Multi-lingual assistance The China-Ireland conference is unique in that most of the delegates attending are either from Universities in Ireland or in China. The campus navigation system described in this paper includes pages in both English and Chinese. Figure 6 shows a screenshot of the campus navigation system interface provided in the Chinese language. We have incorporated the Chinese language to the system which includes the turn-by-turn description for 


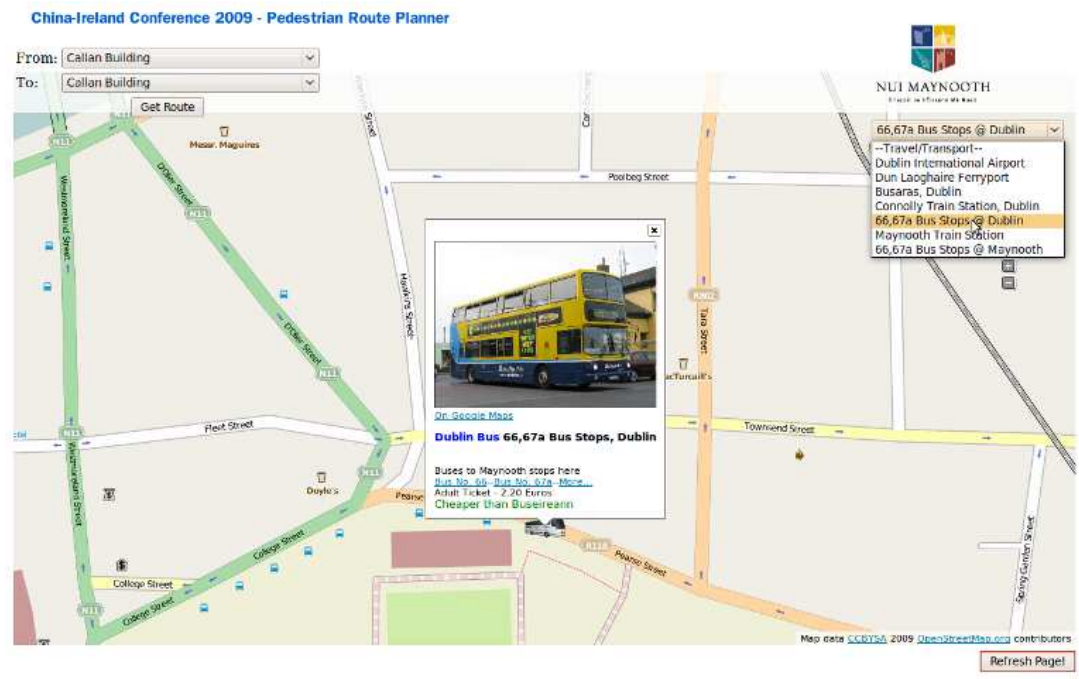

Fig. 5: The Dublin Bus travel information appears in the marker infowindow

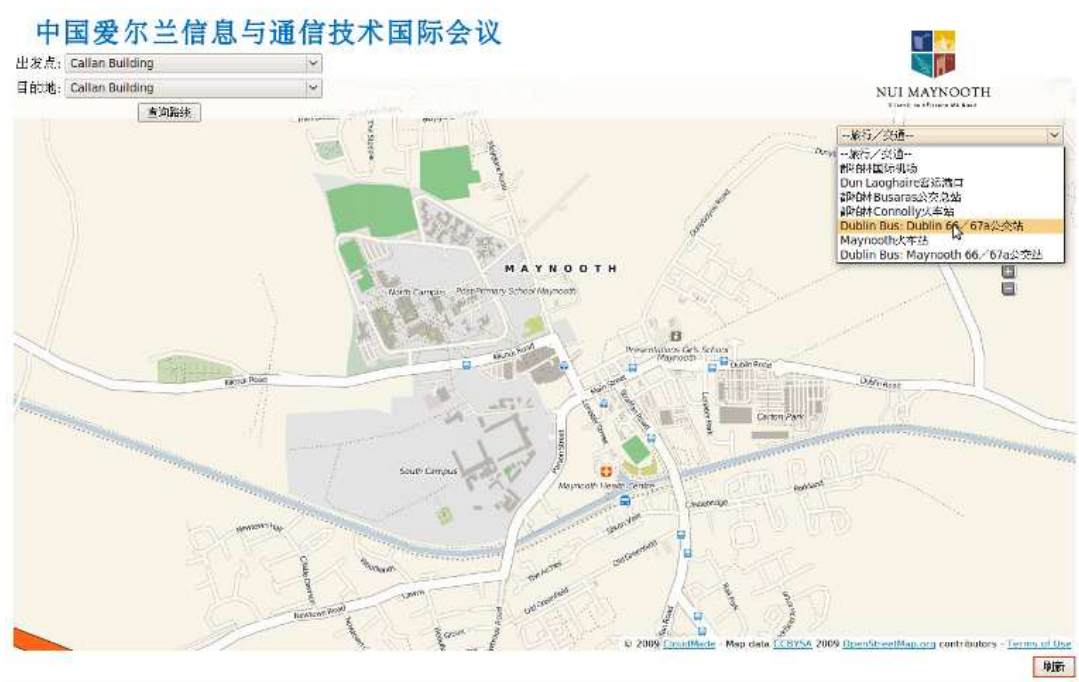

Fig. 6: A screenshot of the campus navigation system interface provided in the Chinese language 
routing and POI. In the future we also intend to include Irish, French, German, and Polish languages as the language options.

\section{Conslusions and future work}

Cloudmade Web Lite API is still in an early stage of development. One of the problems with this "early adopters" stage is that there is little documentation to help developers working with the API. We see much potential in how we can customise the use of OSM data using the CloudMade Web Lite API. The present work designs a multi-lingual campus guidance system solution for international conferences in NUIM using map data from OpenStreetMap. It aims to be a common tool for any international conferences on the same campus. In this paper we demonstrated a prototype solution for the CIICT 2009 conference on NUIM campus. Currently it uses 2D maps for the guidance system. Images provide geovisual assistance at present. Consequently we intend to build a campus navigation system which is context aware and to make this user interface reusable by changing the schema and by making it customizable by any department in the university. The larger research cluster we are working in has built a 3D model of the campus. In future work the extension of the campus navigation system with $3 \mathrm{D}$ visualisation inside and outside buildings will be investigated. Figure 7 shows a sample of the 3D model for the NUIM campus. It is hoped that this 3D extension will be provided also for mobile users in the near future.

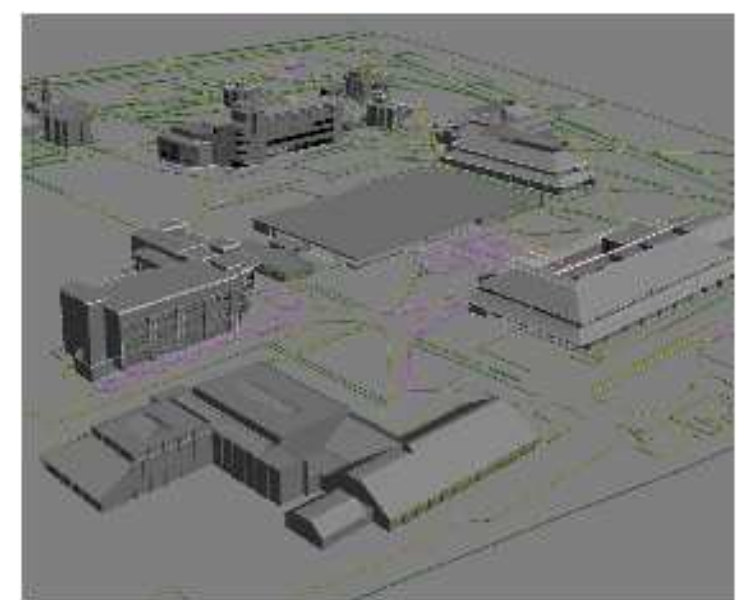

Fig. 7: An example of the 3D model of the NUIM campus [34] 


\section{Acknowledgements}

Research presented in this paper was funded by a Strategic Research Cluster grant (07/SRC/I1168) by Science Foundation Ireland under the National Development Plan. The authors gratefully acknowledge this support. Part of this work is funded by the Environmental Protection Agency STRIVE research programme under the National Development Plan. The assistance of ZhanGang Zhang (Department of Computer Science) is acknowledged for providing us with the Chinese translations of various places and features in Maynooth and Dublin.

\section{References}

1. Changbin Yu, X.X.: Campus navigation system for new students based on .net technology. Journal of Fujian Computer 10 (2008) 121-122

2. U Baaser, M.L.G.S.H.D.H.N.K.R.L.G.B.: Campusgis of the university of cologne: a tool for orientation, navigation, and management. Proceedings of Geoinformatics Proc of SPIE 6421 (2006)

3. Guangchen $\mathrm{Wu}$, Y.L.: Research into the digital campus navigation system based on arcgis engine. Journal of Liaoning Technology College 11 (2009) 12-13 Issue 1.

4. Wen Guangchao, Deng Yinsheng, Y.Y.X.J.Y.M.: Design and realization of campus navigation system for henan polytechnic university. Journal of Geomatics 33 (April 2008) 38-40

5. Robert Workman, A.G.J.L.C.: Campus google map applications. EB/OL (2005)

6. Yang Yang, J.Z.: Developing campus information service system based on googlemaps. In: Proceedings of the 6th Seminar on Cartography and GIS of China, Urumqi, Xinjiang China (2008) 555-562

7. UCL: UCL Campus Route Finder. http://crf.casa.ucl.ac.uk/ - last accessed, July 2009 (2009)

8. Huiling Guo, Yu Liu, Y.C.: The research and implementation of 3d digital campus navigation system. Journal of Shandong University of Technology (Natural Science Edition) 22(5) (2008) 30-32

9. Luo Dan, T.G.W.L.Z.S.: A study for 3 d virtual campus navigation system based on gis. In: Proceedings of 4 th International Conference on Wireless Communications. Volume Networking and Mobile Computing. (2008)

10. Al Takrouri B., Canonico A., G.L.J.M.T.C.S.A.: eyejot - a ubiquitous contextaware campus information system. In: Proceedings of 2nd International Conference on Pervasive Computing and Applications. (2007)

11. Fengyuan Xu, Z.F.: Campus gis information system supported by p2p. In: Proceedings of the 2007 International Symposium on Applications and the Internet Workshops (SAINTW'07). (2007)

12. Jia Nong, Tinglei Huang, Z.C.: Research on soa for campus information system model. In: Proceedings of 2008 International Conference on Computer Science and Software Engineering. (2008) 824-827

13. Lei Cai, Zhihui Liu, J.Z.: Query system design and implement based on webgis. In: Proceedings of the 6th Seminar on Cartography and GIS of China, Urumqi, Xinjiang China (2008) 241-248

14. Strachan, S.: Gpstunes: controlling navigation via audio feedback. Number 159593-089-2 (2005) $275-278$ 
15. GoogleMM: Google Maps for Mobile. http://www.google.com/mobile/products/ maps.html - last accessed, July 2009 (2009)

16. Nokia: Nokia Maps. http://europe.nokia.com/explore-services/maps/ download - last accessed, July 2009 (2009)

17. Beeharee, A.K.: A natural wayfinding exploiting photos in pedestrian navigation systems. Number 1-59593-390-5 (2006) $81-88$

18. Ertan, S. Lee, C.W.A.T.H.P.A.: A wearable haptic navigation guidance system. Number 0-8186-9074-7 (2002) 164-165

19. Wasinger, R.: Human-Computer Interaction with Mobile Devices and Services. Volume Volume 2795/2003. Springer Berlin / Heidelberg (2003)

20. ProgrammableWeb: Programmable Web. http://www.programmableweb.com/ last accessed, July 2009 (2009)

21. Google: Google Maps API. http://code.google.com/apis/maps/ - last accessed, July 2009 (2009)

22. Yahoo: Yahoo Maps API. http://developer.yahoo.com/maps/ - last accessed, July 2009 (2009)

23. OSM: Open Street Map. http://www.openstreetmap.org/ - last accessed, July 2009 (2009)

24. Microsoft: Bing Maps API. http://www.microsoft.com/maps/developers/ - last accessed, July 2009 (2009)

25. GoogleMapMaker: Google MapMaker. http://www.google.com/mapmaker - last accessed, July 2009 (2009)

26. Wikimapia: Wikimapia. http://wikimapia.org/ - last accessed, July 2009 (2009)

27. Haklay, M.M., Weber, P.: Openstreetmap: User-generated street maps. PERVASIVE computing (August 2008) 12-18

28. Bloch, J.: How to design a good api and why it matters. Technical report, Google Inc. (2006)

29. CIICT: CIICT Webpage. http://www . ciict.org/ - last accessed, July 2009 (2009)

30. CloudMade: CloudMade Web Maps Lite API. http://developers.cloudmade. com/projects/show/web-maps-lite - last accessed, July 2009 (2009)

31. Eckert, C.: GPS Babel

32. CGEnglish: Campus Guidance - English. http://ciict.cs.nuim.ie/files/ location/MaynoothEn.html - last accessed, July 2009 (2009)

33. Hile, H., Vedantham, R., Cuellar, G., Liu, A.L., Gelfand, N., Grzeszczuk, R., Borriello, G.: Landmark-based pedestrian navigation from collections of geotagged photos. In Wiberg, M., Zaslavsky, A.B., eds.: MUM, ACM (2008) 145-152

34. Jianghua Zheng, B Ciepłuch, P.M.A.W.: Location based services of university town based on openstreetmap: Nui maynooth as an example. In: to be published by Proceedings of CIICT2009. (2009) 